\section{A Recursive Algorithm for Maximum Likelihood-Based Identification of Blur from Multiple Observations}

\author{
A. N. Rajagopalan and Subhasis Chaudhuri
}

\begin{abstract}
A maximum likelihood-based method is proposed for blur identification from multiple observations of a scene. When the relations among the blurring functions are known, the estimate of blur obtained using the proposed method is very good. Since direct computation of the likelihood function becomes difficult as the number of images increases, we propose an algorithm to compute the likelihood function recursively.
\end{abstract}

Index Terms-Blur identification, Gaussian defocus, ML estimator, multiple observations, recursive computation.

\section{INTRODUCTION}

Blur identification is a very important step toward restoration, and in actual practice must be estimated from the degraded image itself. The earliest work on blur identification concentrated on point spread functions (psf's), Fourier transforms of which have zeros on the unit bicircle [1]. In more recent works, the original image is modeled as an autoregressive (AR) process and the identification problem is formulated as a maximum likelihood (ML) estimation problem [2]-[8]. An overview of the developments in image and blur identification under the ML framework is given in [7]. Recently, the recovery of an image from multiple observations of it has been receiving much attention. In [9], Katsaggelos et al. suggest an algorithm that incorporates multiple distorted versions of a signal and results in a restoration error approaching zero with few iterations. In [10], Ghiglia develops a scheme for image restoration from multiple, blurred images based on the constrained least squares approach. Ward [11] considers restoration from differently blurred versions of an image in the presence of noise. However, in all these methods the psf is either exactly or partially known.

In this correspondence, we propose an ML-based blur identification method that uses multiple, blurred versions of the original image to get improved estimates of blur. When the relations among the blurring functions are known, we show that the estimate of blur obtained by using the proposed method is very good [12]. The improvement is particularly significant under severe blurring conditions. We select the problem of blurring due to defocusing [13] as an application to demonstrate the usefulness of the proposed technique. The degree of improvement in the estimate of blur depends on the relative blurring among the images. However, with an increase in the number of blurred images, the size of the cross-correlation matrix increases and direct computation of the likelihood function becomes cumbersome. Further, the computation has to start afresh for each additional observation of the scene. To tackle this, we propose an elegant method that computes the likelihood function recursively as more observations are added.

Manuscript received May 22, 1996; revised August 12 1997. The associate editor coordinating the review of this manuscript and approving it for publication was Dr. Jun Zhang.

The authors are with the Department of Electrical Engineering, Indian Institute of Technology, Bombay 400 076, India (e-mail: raju@ee.iitb.ernet.in; sc@ee.iitb.ernet.in).

Publisher Item Identifier S 1057-7149(98)04372-3.

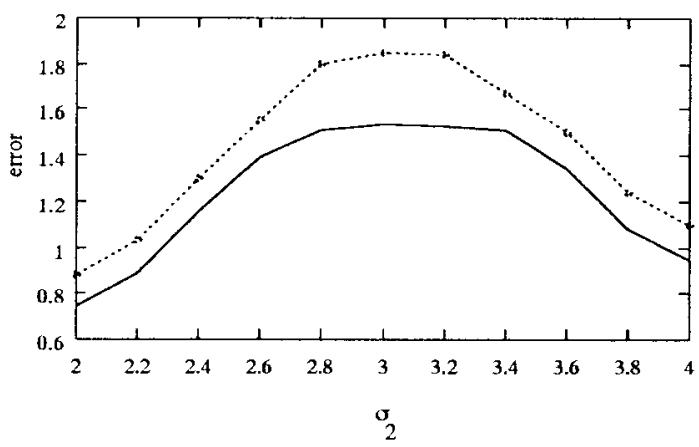

Fig. 1. Magnitude of the error in the estimate of $\sigma_{1}$ for various values of $\sigma_{2}$. The continuous and the dotted lines correspond to SNR of 40 and 10 $\mathrm{dB}$, respectively.

\section{ML Blur Identification from Multiple Images}

We model the discrete original image $f(i, j)$ by a two-dimensional (2-D) AR process with causal support and coefficients $a(i, j)$, driven by a zero mean homogeneous Gaussian white noise process $v(i, j)$ with variance $\sigma_{v}^{2}$. The observed image $g(i, j)$ is modeled as the output of a 2-D linear space-invariant system characterized by the psf $h(i, j)$. The observation noise $w(i, j)$ is assumed to be an additive zero-mean white Gaussian process with variance $\sigma_{w}^{2}$. Hence, in the Fourier domain, we have $\bar{F}=\left(I-\Lambda_{A}\right)^{-1} \bar{V}$ and $\bar{G}=\Lambda_{H} \bar{F}+\bar{W}$, where $\bar{F}, \bar{G}, \bar{V}$, and $\bar{W}$ are the discrete Fourier transforms (DFT's) of the raster-scanned sequences $f(i, j), g(i, j), v(i, j)$, and $w(i, j)$, respectively. Matrices $\Lambda_{A}$ and $\Lambda_{H}$ are diagonal with entries that correspond to the DFT's of the sequences $a(i, j)$ and $h(i, j)$, respectively.

The following relationships are derived for one-dimensional (1-D) signals. Extension to the 2-D case is straightforward. Given $M$ differently blurred versions of the original image, we have

$$
\bar{G}^{i}=\Lambda_{H_{i}} \bar{F}+\bar{W}^{i}, \quad i=1,2, \cdots, M .
$$

Notationally, the bar in $\bar{G}^{i}$ represents the process while $G^{i}$ is a realization of the process. Noise processes $\bar{W}^{k}$ and $\bar{W}^{l}$ are assumed to be statistically independent for $k \neq l$. Let $\bar{G}_{M}=$ $\left[\begin{array}{llll}G^{1^{T}} & G^{2^{T}} & \cdots & \bar{G}^{M^{T}}\end{array}\right]^{T}$ and $G_{M}=\left[\begin{array}{llll}G^{1^{T}} & G^{2^{T}} & \cdots & G^{M^{T}}\end{array}\right]^{T}$. Here, " $T$ " represents transpose. It is straightforward to show from (1) that $\bar{G}_{M}$ is also jointly Gaussian. Therefore, the probability density function of $\bar{G}_{M}$ is given by

$$
p\left(G_{M}\right)=\frac{1}{(2 \pi)^{M N / 2}\left(\operatorname{det} P_{M}\right)^{1 / 2}} \exp \left(-\frac{1}{2} G_{M}^{H} P_{M}^{-1} G_{M}\right)
$$

where

$$
\begin{aligned}
P_{M} & =E\left[\bar{G}_{M} \bar{G}_{M}^{H}\right] \\
& =\left[\begin{array}{cccc}
P^{1,1} & P^{1,2} & \cdots & P^{1, M} \\
P^{2,1} & P^{2,2} & \cdots & P^{2, M} \\
\vdots & \vdots & \vdots & \vdots \\
P^{M, 1} & P^{M, 2} & \cdots & P^{M, M}
\end{array}\right] .
\end{aligned}
$$

$E$ is the expectation operator, $N$ is the number of samples, and $P^{i, j}=E\left[\bar{G}^{i} \bar{G}^{j H}\right]$. Here, " $H$ " represents the Hermitian. It can be shown that $P^{i, j}=\sigma_{v}^{2} \Lambda_{H_{i}}\left(I-\Lambda_{A}\right)^{-1}\left(I-\Lambda_{A}\right)^{-H} \Lambda_{H_{j}}^{H}+\sigma_{w}^{2} \delta_{i, j} I$, 


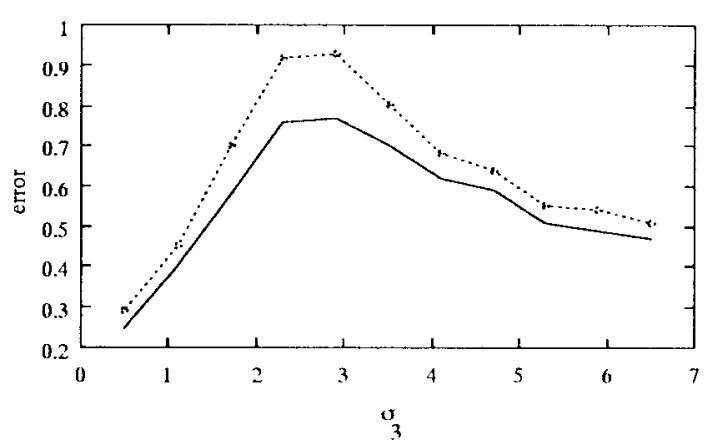

(a)

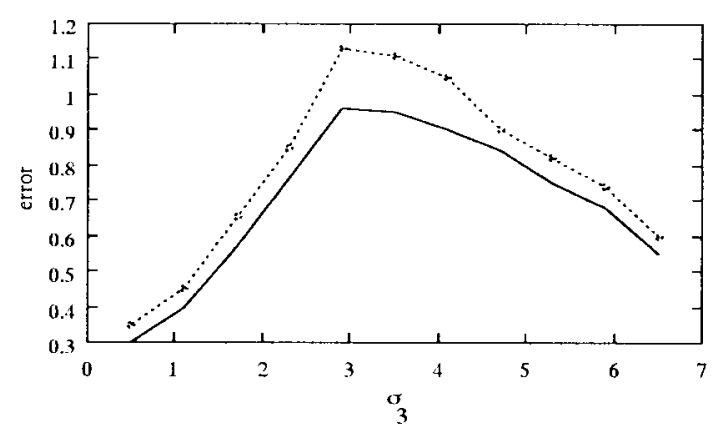

(b)

Fig. 2. Magnitude of the error in the estimate of $\sigma_{1}$ for various values of $\sigma_{3}$ and for (a) $\sigma_{2}=2.0$ and (b) $\sigma_{2}=4.0$. The continuous and the dotted lines correspond to SNR of 40 and $10 \mathrm{~dB}$, respectively.

where $P^{i, j}$ is a diagonal matrix whose $k$ th entry is given by

$$
P^{i, j}(k, k)=\frac{H_{i}(k) H_{j}^{*}(k)}{|1-A(k)|^{2}} \sigma_{v}^{2}+\sigma_{w}^{2} \delta_{i, j}, \quad k=0,1, \cdots, N-1 .
$$

Here, $H_{i}(k)$ and $A(k)$ are the DFT's of $h_{i}(m)$ and $a(m)$ while "*” represents complex conjugate.

Let the unknown parameters be denoted by the vector $\theta=$ $\left\{a(m), h_{1}(m), \cdots, h_{M}(m), \sigma_{w}^{2}, \sigma_{v}^{2}\right\}$ and $h_{i}(m)$ be the psf corresponding to the blurring in the $i$ th observation. The blur identification problem focuses on estimating the unknown parameters $h_{i}(m)$, $i=1, \cdots, M$ from the $M$ noisy and blurred observations. The ML estimation problem of $\theta$ can now be expressed as

$$
\min _{\theta} F_{M}(\theta) \text { where } F_{M}(\theta)=\log \left(\operatorname{det} P_{M}\right)+G_{M}^{H} P_{M}^{-1} G_{M} \text {. }
$$

Equation (5) specifies a complicated nonlinear optimization problem in several variables mainly because of the nonquadratic behavior of $\log$ det $P_{M}$. To obtain a unique solution, additional constraints about the unknown parameters need to be incorporated.

\section{RECURSIVE COMPUTATION OF THE LiKELIHOOD FunCTION}

To compute $F_{M}(\theta)$, direct evaluation of det $P_{M}$ and $P_{M}^{-1}$ in (5) would be very cumbersome for increasing $M$. In this section, we propose an elegant method that recursively computes $F_{M}(\theta)$. The method uses some important results from linear algebra [14].

From (3), the matrix $P_{M}$ can be rewritten in partitioned form as

$$
P_{M}=\left[\begin{array}{cc}
P_{M-1} & D_{M} \\
D_{M}^{H} & P^{M, M}
\end{array}\right]
$$

where $D_{M}=\left[P^{1, M} P^{2, M} \cdots P^{M-1, M}\right]^{T}$. It may be noted that all $P^{i, j} \mathrm{~S}$ are diagonal, and further, $P^{i, i}$ is real. Therefore

$$
P_{M}^{-1}=\left[\begin{array}{ll}
A_{M} & B_{M} \\
B_{M}^{H} & C_{M}
\end{array}\right]
$$

where

$$
\begin{aligned}
& C_{M}=\left(P^{M, M}-D_{M}^{H} P_{M-1}^{-1} D_{M}\right)^{-1} \\
& B_{M}=-P_{M-1}^{-1} D_{M} C_{M} \\
& A_{M}=P_{M-1}^{-1}+P_{M-1}^{-1} D_{M} C_{M} D_{M}^{H} P_{M-1}^{-1} .
\end{aligned}
$$

The determinant of $P_{M}$ can be rewritten as

$$
\text { det } \begin{aligned}
P_{M} & =\left(\operatorname{det} P_{M-1}\right) \operatorname{det}\left(P^{M, M}-D_{M}^{H} P_{M-1}^{-1} D_{M}\right) \\
& =\left(\operatorname{det} P_{M-1}\right) \operatorname{det}\left(C_{M}^{-1}\right) .
\end{aligned}
$$

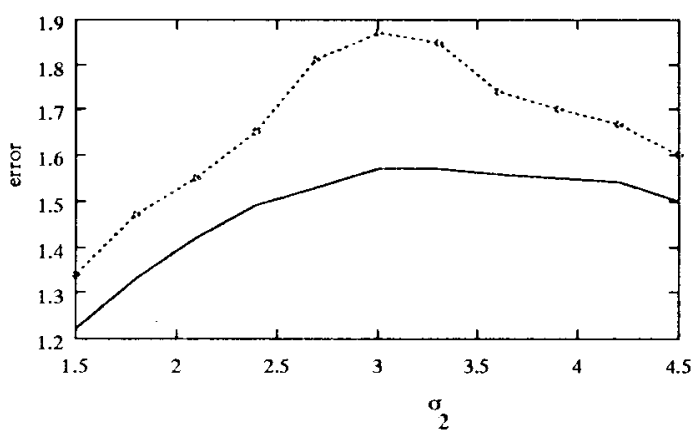

Fig. 3. Magnitude of the error in the estimate of $\sigma_{1}$ for various values of $\sigma_{2}$ for the cameraman image. The continuous and the dotted lines correspond to SNR of 40 and $10 \mathrm{~dB}$, respectively.

TABLE I

FOCUSING RANGE AND ERROR IN THE Estimate of Depth For the SCENE IN Fig. 6

\begin{tabular}{c|c|c|c}
\hline $\begin{array}{c}\text { First } \\
\text { image }\end{array}$ & $\begin{array}{c}\text { Second } \\
\text { image }\end{array}$ & $\begin{array}{c}\text { Third } \\
\text { image }\end{array}$ & $\%$ Error \\
\hline \multirow{4}{*}{$140 \mathrm{~cm}$} & - & - & 13.10 \\
\cline { 2 - 4 } & $180 \mathrm{~cm}$ & - & 9.35 \\
& $180 \mathrm{~cm}$ & $300 \mathrm{~cm}$ & 4.70 \\
\cline { 2 - 4 } & $110 \mathrm{~cm}$ & - & 8.50 \\
& $110 \mathrm{~cm}$ & $90 \mathrm{~cm}$ & 2.50 \\
\hline
\end{tabular}

Using (7) and (8) in (5), we get the important recursive relation

$$
\begin{aligned}
F_{M}(\theta)= & F_{M-1}(\theta)+\log \operatorname{det} C_{M}^{-1} \\
& +G_{M-1}^{H} P_{M-1}^{-1} D_{M} C_{M} D_{M}^{H} P_{M-1}^{-1} G_{M-1} \\
& +G_{M-1}^{H} B_{M} G^{M}+G^{M} B_{M}^{H} G_{M-1}+G^{M^{H}} C_{M} G^{M} .
\end{aligned}
$$

Since $P_{1}=P^{1,1}$ is a diagonal matrix and so is $C_{M}$ (for all $M$ ), no inversion is required in the proposed recursive formulation.

We now proceed to compute the likelihood function for $M=1,2$ and for the general case of $M$ images.

\section{A. Computation of $F_{1}(\theta)$}

From (5) $F_{1}(\theta)=\log \operatorname{det} P_{1}+G_{1}^{H} P_{1}^{-1} G_{1}=$ $\sum_{k=0}^{N-1} \log \left[P^{1,1}(k, k)\right]+\left[\left|G^{1}(k)\right|^{2}\right] /\left[P^{1,1}(k, k)\right]$. The exact expression for $P^{1,1}(k, k)$ that relates to the parameter $\theta$ is given by (4). The above expression is exactly the same as the one derived 

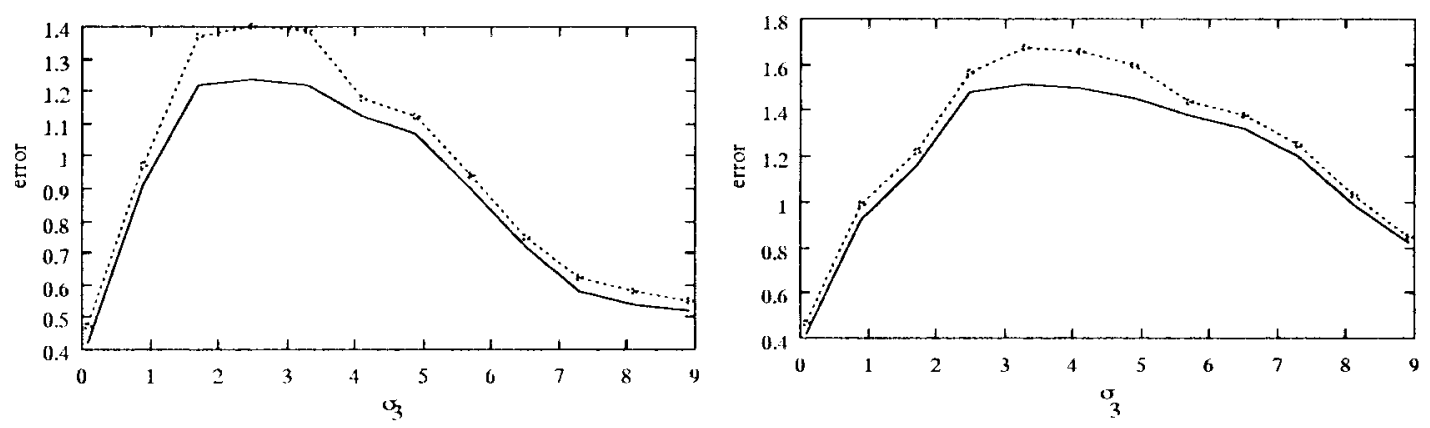

Fig. 4. Magnitude of the error in the estimate of $\sigma_{1}$ for different values of $\sigma_{3}$ for the cameraman image and for (a) $\sigma_{2}=1.5$ and (b) $\sigma_{2}=4.5$. The continuous and the dotted lines correspond to SNR of 40 and $10 \mathrm{~dB}$, respectively.

in [7] for the case of a single image. Thus, our formulation provides a general framework in which $M=1$ is a special case.

\section{B. Computation of $F_{2}(\theta)$}

Using (9) and the fact that the matrices involved in the computation are all diagonal, it can be shown that

$$
\begin{aligned}
F_{2}(\theta)= & F_{1}(\theta)+\sum_{k=0}^{N-1} \log \left[P^{2,2}(k, k)-\frac{\left|P^{2,1}(k, k)\right|^{2}}{P^{1,1}(k, k)}\right] \\
& +C_{2}(k, k)\left|G^{1}(k) \frac{P^{2,1}(k, k)}{P^{1,1}(k, k)}-G^{2}(k)\right|^{2}
\end{aligned}
$$

where

$$
C_{2}(k, k)=\frac{1}{\left[P^{2,2}(k, k)-\frac{\left|P^{2,1}(k, k)\right|^{2}}{P^{1,1}(k, k)}\right]}
$$

\section{Computation of $F_{M}(\theta)$}

One may continue proceeding as in Section III-B to obtain the general terms in the expression for $F_{M}(\theta)$. However, the expression becomes unwieldy beyond $M=2$. Here we provide the steps involved in computing the likelihood function recursively.

Step 1) Initialize $F_{1}(\theta)=\log \operatorname{det} P_{1}+G_{1}^{H} P_{1}^{-1} G_{1}$, where $P_{1}=P^{1,1}$ is given by (4). Minimize $F_{1}(\theta)$ to obtain the estimate $\theta=\theta_{1}$ and set $M=2$.

Step 2) Obtain $C_{M}=\left(P^{M, M}-D_{M}^{H} P_{M-1}^{-1} D_{M}\right)^{-1}$.

Step 3) Get $B_{M}=-P_{M-1}^{-1} D_{M} C_{M}$.

Step 4) Use FFT to obtain $G^{M}$ from the $M$ th observation.

Step 5) Compute $F_{M}(\theta)=F_{M-1}(\theta)+\log \operatorname{det} C_{M}^{-1}+$ $G_{M-1}^{H} P_{M-1}^{-1} D_{M} C_{M} D_{M}^{H} P_{M-1}^{-1} G_{M-1}+$ $G_{M-1}^{H} B_{M} G^{M}+G^{M^{H}} B_{M}^{H} G_{M-1}+G^{M^{H}} C_{M} G^{M}$.

Step 6) Minimize $F_{M}(\theta)$ using $\theta_{M-1}$ as the initial estimate and obtain $\theta_{M}$.

Step 7) $M \leftarrow M+1$ and go to Step 2 .

\section{A SPECial Application}

Point spread functions of degradations encountered in practice may have a support of considerable extent. Without additional knowledge about the relations between the psf coefficients, this requires the estimation of a large number of independent parameters. Low-order parametric blur models when incorporated into the identification scheme make the identification algorithm applicable to more realistic blurs and improve the identification results. An additional advantage of these parametric models is that they can be initialized more easily than the nonparametric ones. Even after substituting the selected parametric blur model, the nonlinear optimization problem involving

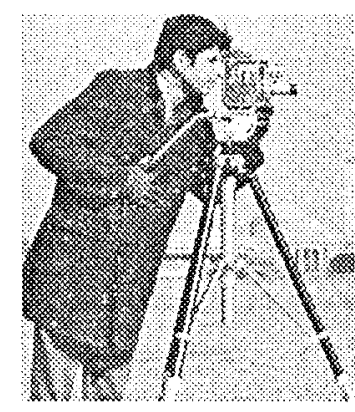

(a)

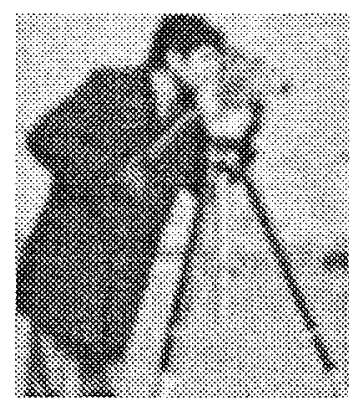

(c)

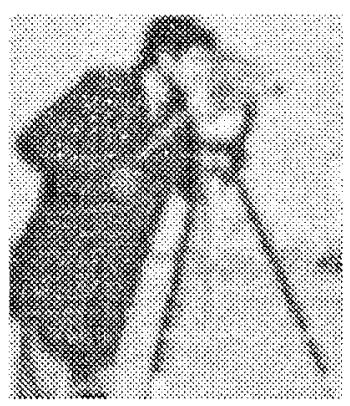

(b)

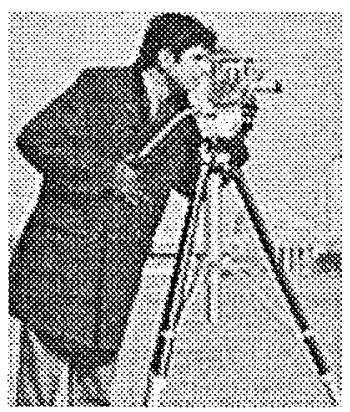

(d)
Fig. 5. (a) Original cameraman. (b)-(d) Restored images corresponding to $\hat{\sigma}_{1}=1.7,2.1$, and 2.6, respectively, using the Wiener filter. The SNR was $40 \mathrm{~dB}$.

(9) would rarely have an explicit solution. In general, it must be solved by numerical methods, which can now be done efficiently because of the low dimensionality of the problem.

A priori information about the psf is available in many cases of interest. For example, in the scheme on recovery of depth from defocused images, the camera blur is usually modeled as a Gaussian function $h_{m}(i, j)=\left(1 / 2 \pi \sigma_{m}^{2}\right) \exp \left[-\left(i^{2}+j^{2}\right) / 2 \sigma_{m}^{2}\right], m=$ $1,2, \cdots, M$. The blurred image of the scene is given by the convolution of the original focused image and the psf of the system corresponding to that depth. The blur parameter $\sigma_{m}$ depends on the lens parameters and the depth $D$ and is given by $\sigma_{m}=$ $\rho r_{m} v_{m}\left(1 / F_{l_{m}}-1 / v_{m}-1 / D\right)$ where $F_{l_{m}}, v_{m}$, and $r_{m}$ are the focal length, the image plane-to-lens distance, and the lens aperture, respectively, for the $m$ th camera setting. For different lens settings, the blur parameters corresponding to different blurred images are related [15] by $\sigma_{1}=\alpha_{i} \sigma_{i}+\beta_{i}, i=2, \cdots, M$, where $\alpha_{i}=$ $r_{1} v_{1} / r_{i} v_{i}$ and $\beta_{i}=r_{1} v_{1}\left(1 / F_{l_{1}}-1 / v_{1}-1 / F_{l_{i}}+1 / v_{i}\right)$ are known constants. The relative blurring between $\sigma_{1}$ and $\sigma_{i}$ is governed by $\alpha_{i}$ and $\beta_{i}$. Given the relation among the blurring functions and the 


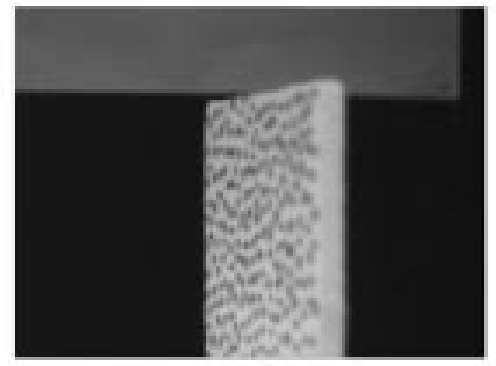

(a)

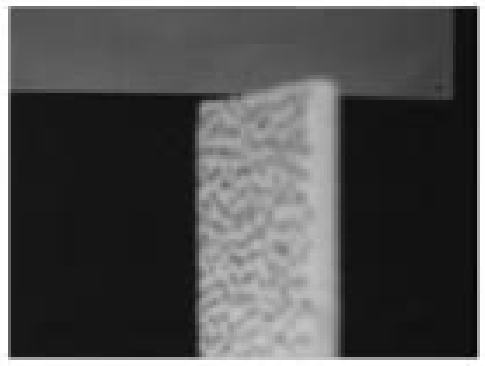

(b)

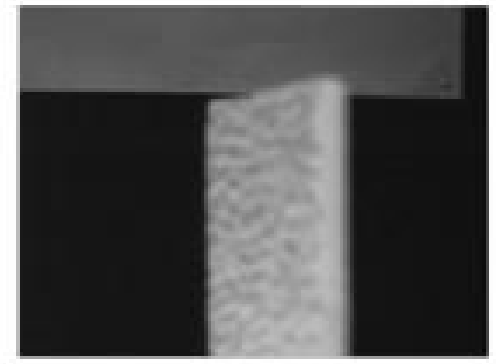

(c)

Fig. 6. Some of the defocused images in the experimental setup. Images correspond to focusing range of (a) $140 \mathrm{~cm}$, (b) $180 \mathrm{~cm}$, and (c) $300 \mathrm{~cm}$.

corresponding defocused and noisy images, the problem boils down to estimating $\sigma_{1}$.

\section{EXPERIMENTAL RESULTS}

The blurring function was chosen to be Gaussian in our experimental studies. Given $M$ number of observations, $F_{M}(\theta)$ was minimized with respect to the unknown parameter set $\theta$ using the steepest descent method. The noise was assumed to be zero-mean, white Gaussian and SNR's of 40 and $10 \mathrm{~dB}$ were considered. In the first set of simulations, an arbitrary 1-D signal of length 64 samples was blurred severely by a Gaussian function of standard deviation $\sigma_{1}=3.0$. The order of the AR model for the original signal was chosen to be two. The initial value for the estimate of $\sigma_{1}$ was taken as 1.0. The estimates of $\sigma_{1}$ obtained by minimizing $F_{1}(\theta)$ were 1.46 and 1.16 for SNR of 40 and $10 \mathrm{~dB}$, respectively. About 50 iterations were required for convergence. The estimates obtained by using a single image turned out to be quite poor, as expected. We next generated a second blurred signal for different values of $\sigma_{2}$. The estimate of $\sigma_{1}$ (denoted by $\hat{\sigma}_{1}$ ) was now obtained by minimizing $F_{2}(\theta)$ and the magnitude of the error, defined as $\left|\sigma_{1}-\hat{\sigma}_{1}\right|$, is plotted in Fig. 1 . About 40 iterations were now required for convergence. We observe that there is a substantial improvement in the estimate of $\sigma_{1}$ when the second blurred signal is relatively either more focused (i.e., $\sigma_{2} \ll \sigma_{1}$ ) or more blurred (i.e., $\sigma_{2} \gg \sigma_{1}$ ) compared to the first signal. This can also be proved by showing the corresponding Cramér-Rao bound to be a decreasing function in terms of relative blurring [16]. The estimate of $\sigma_{1}$ is more immune to noise as the relative blurring increases. Similar observations were also made by Ghiglia [10] in the context of image restoration from multiple observations. A third blurred signal was next generated and the errors in the estimate of $\sigma_{1}$ are plotted in Fig. 2(a) and (b). The algorithm converged in 25 iterations itself. From these plots, we again notice that the improvement in the estimate of $\sigma_{1}$ (and its immunity to noise) are quite significant when the third signal is relatively more focused or blurred than the first two signals. In the second set of simulation results, the MIT cameraman image was severely blurred by a 2-D Gaussian blur with $\sigma_{1}=3.0$. The order of the AR model was chosen to be two. The initial estimate of $\sigma_{1}$ was taken as 1.0. The ML estimate of $\sigma_{1}$ obtained by using only this blurred image was poor, and was 1.42 and 1.14 for SNR of 40 and $10 \mathrm{~dB}$, respectively. About 50 iterations were needed for convergence. Next, we generated a second blurred image and the errors in the ML estimates of $\sigma_{1}$ corresponding to different values of $\sigma_{2}$ are plotted in Fig. 3. The algorithm now converged in 40 iterations. We again note that the estimate of $\sigma_{1}$ improves (and so does its immunity to noise) when $\sigma_{2}$ is either small or large as compared to $\sigma_{1}$. Similar observations were noted when a third blurred image was used [Fig. 4(a) and (b)]. Only 25 iterations were now needed for convergence. The original and the Wiener-filter restored images corresponding to the estimates of $\sigma_{1}$ obtained with one, two, and three observations are shown in Fig. 5. It is clear that an improved estimate of $\sigma_{1}$ results in a significant improvement in the restored image.

Finally, for testing the performance of the proposed method on real blurs, a scene was constructed using a planar object whose farthest point was at a distance of $100 \mathrm{~cm}$, while the nearest point was about $80 \mathrm{~cm}$ from the camera (Fig. 6). The depth $D$ corresponding to the nearest end of the object was estimated using a local window. From Table I, we observe that the estimate of the depth improves as more observations are used. The estimate of the depth is quite good when the successive observation is better focused than the previous ones. The above procedure can be continued by incorporating even more number of observations to improve the estimate of depth.

\section{CONCLUSIONS}

In this paper, an ML-based blur identification method that uses multiple blurred versions of the original image has been proposed. We have shown that the method gives significant improvement in the estimate of blur for the class of Gaussian blurs, more so when blurring is severe. We have also proposed a scheme to find the likelihood function recursively because direct computation becomes difficult with increasing number of images. The degree of improvement in the estimate of the blur is better as the relative blurring increases. Also, the estimate of blur becomes more resistant to noise. The proposed method can be used in many practical computer vision applications like depth from defocus and electron microscopy. We are currently working on extending the performance analysis of the method to other classes of blur.

\section{ACKNOWLEDGMENT}

The authors thank the anonymous reviewers for their useful criticism and suggestions.

\section{REFERENCES}

[1] H. C. Andrews and B. R. Hunt, Digital Image Restoration. Englewood Cliffs, NJ: Prentice-Hall, 1977.

[2] A. M. Tekalp, H. Kaufman, and J. W. Woods, "Identification of image and blur parameters for the restoration of noncausal blurs," IEEE Trans. Acoust., Speech, Signal Processing, vol. ASSP-34, pp. 963-972, Aug. 1986.

[3] R. L. Lagendijk, A. K. Katsaggelos, and J. Biemond, "Iterative identification and restoration of images," in Proc. IEEE Int. Conf. Acoustics, Speech, Signal Processing, New York, 1988, pp. 992-995. 
[4] K. T. Lay and A. K. Katsaggelos, "Image identification and restoration based on the expectation-maximization algorithm," Opt. Eng., vol. 29 , pp. 436-445, May 1990.

[5] R. L. Lagendijk, J. Biemond, and D. E. Boekee, "Identification and restoration of noisy blurred images using the expectation-maximization algorithm," IEEE Trans. Acoust., Speech, Signal Processing, vol. 38, pp. 1180-1191, July 1990.

[6] _ "Hierarchial blur identification," in Proc. IEEE Int. Conf. Acoust., Speech, Signal Processing, 1990, pp. 1889-1892.

[7] R. L. Lagendijk, A. M. Tekalp, and J. Biemond, "Maximum likelihood image and blur identification: A unifying approach," Opt. Eng., vol. 29, pp. 422-435, May 1990.

[8] J. Kim and J. W. Woods, "Image identification and restoration in the sub-band domain," IEEE Trans. Image Processing, vol. 3, pp. 312-314, May 1994.

[9] A. K. Katsaggelos and R. W. Schafer, "Iterative deconvolution using several different distorted versions of an unknown signal," in Proc. IEEE Int. Conf. Acoustics, Speech, Signal Processing, Boston, MA: 1983, pp. 659-662.

[10] D. C. Ghiglia, "Space-invariant deblurring given $N$ independently blurred images of a common object," J. Opt. Soc. Amer. A, vol. 1, pp. 398-402, Apr. 1984.

[11] R. K. Ward, "Restoration of differently blurred versions of an image with measurement errors in the PSF's," IEEE Trans. Image Processing, vol. 2, pp. 369-381, July 1993.

[12] A. N. Rajagopalan and S. Chaudhuri, "Maximum likelihood estimation of blur from multiple observations," in Proc. IEEE Int. Conf. Acoustics, Speech, Signal Processing, Munich, Germany, Apr. 1997, pp. $2577-2580$.

[13] M. Subbarao, "Efficient depth recovery through inverse optics," in Machine Vision for Inspection and Measurement, H. Freeman, Ed. New York: Academic, 1989

[14] F. A. Graybill, An Introduction to Linear Statistical Models. New York: McGraw-Hill, 1961, vol. 1.

[15] A. N. Rajagopalan and S. Chaudhuri, "Space-variant approaches to the recovery of depth from defocused images," Computer Vision and Image Understanding, to be published.

[16] _ _ "Optimal selection of camera parameters for recovery of depth from defocused images," in Proc. IEEE Int. Conf. Computer Vision and Pattern Recognition, U.S. Virgin Islands, June 1997.

\section{Region Growing: A New Approach}

\author{
S. A. Hojjatoleslami and J. Kittler
}

Abstract-A new region growing method for finding the boundaries of blobs is presented. A unique feature of the method is that at each step, at most one pixel exhibits the required properties to join the region. The method uses two novel discontinuity measures, average contrast and peripheral contrast, to control the growing process.

\section{INTRODUCTION}

The segmentation of regions is an important first step for a variety of image analysis and visualization tasks. There is a wide range of image segmentation techniques in the literature, some considered general purpose and some designed for a specific class of images. Conventional segmentation techniques for monochromatic images

Manuscript received November 5, 1995; revised October 27, 1997. The associate editor coordinating the review of this manuscript and approving it for publication was Prof. Patrick A. Kelly.

The authors are with the Centre for Vision, Speech and Signal Processing, University of Surrey, Guildford, Surrey GU2 5XH, U.K. (e-mail: a.hojjatoleslami@ee.surrey.ac.uk).

Publisher Item Identifier S 1057-7149(98)04449-2. can be categorized into two distinct approaches [3]. One is region based, which relies on the homogeneity of spatially localized features, whereas the other is based on boundary finding, using discontinuity measures. The two methods exploit two different definitions of a region which should ideally yield identical results. Homogeneity is the characteristic of a region and nonhomogeneity or discontinuity is the characteristic of the boundary of a region. Based on one or both of these properties, diverse approaches to image segmentation exhibiting different characteristics have been suggested [1], [2], [4], [8]-[10], [12], [13].

We present here a new idea for region growing by pixel aggregation, which uses new similarity and discontinuity measures. A unique feature of the proposed approach is that in each step at most one candidate pixel exhibits the required properties to join the region. This makes the direction of the growing process more predictable. The procedure offers a framework in which any suitable measurement can be applied to define a required characteristic of the segmented region. We use two discontinuity measurements called average contrast and peripheral contrast to control the growing process. Local maxima of these two measurements identify two nested regions, called the average contrast and the peripheral contrast regions. The method first finds the average contrast boundary of a region, then a reverse test is applied to produce the peripheral contrast boundary.

Like existing procedures, the method proposed in this paper is not universal, but it does appear to have a fairly wide application potential, especially in medical image analysis, where the areas corresponding to a tissue of interest appear as bright/dark objects relative to the surrounding tissues.

The concept of the method is presented in the next two sections. The similarity measure used by the method is presented in Section II. Section III introduces the two different discontinuity measures, peripheral contrast and average contrast, and illustrates their behavior on a Gaussian shape image. The capability of our method is then demonstrated on a set of real images in Section IV, followed by a summary and conclusion in Section V.

\section{Growing Process}

The concept of our method, like that of other region growing methods by pixel aggregation, is to start with a point that meets a detection criterion and to grow the point in all directions to extend the region.

Let us assume that the process starts from an arbitrary pixel. The pixel is labeled as a region that then grows based on a similarity measure. In our approach, a boundary pixel is joined to the current region provided it has the highest grey level among the neighbors of the region. This induces a directional growing such that the pixels of high grey level will be absorbed first. When all the high grey level pixels in the region are absorbed, the process continues by absorbing the boundary pixels with monotonically lower and lower grey levels. When several pixels with the same grey level jointly become the candidates to join the region, the first-come first-served strategy is used to select one of them. This makes the region more compact, particularly in situations where the grey levels of the background or the region pixels are very homogeneous.

In order to monitor the pixels joining the region, a grey-level mapping is generated. The mapping is very similar to the mapping of data points from a high-dimensional feature space onto a sequence which is used in the mode separating (MODESP) procedure for cluster analysis proposed by Kittler [7]. The mapping for a small 